\title{
Suppression of dephasing and phase lapses in the fractional quantum Hall regime
}

\author{
Yehuda Dinaii, ${ }^{1}$ Yuval Gefen, ${ }^{1}$ and Bernd Rosenow ${ }^{2,3}$ \\ ${ }^{1}$ Department of Condensed Matter Physics, The Weizmann Institute of Science, Rehovot 76100, Israel \\ ${ }^{2}$ Physics Department, Harvard University, Cambridge, Massachusetts 02138, USA \\ ${ }^{3}$ Institut für Theoretische Physik, Universität Leipzig, D-04103, Leipzig, Germany
}

\begin{abstract}
A charge fluctuator which is electrostatically coupled to a conducting channel may fully dephase quantum transport through the latter. Here, we address the case where a quantum dot (QD), playing the role of a charge fluctuator, is tunnel-coupled to an additional channel. In the case where the latter may support fractional charge, distinct differences from the integer case arise: Abrupt phase lapses of the transmission through the conducting channel occur (which may or may not be equal to $\pi$ ). This is accompanied by a cusp-like suppression of the interferometer's visibility, yet no full dephasing. We interpret our findings in terms of the entanglement between the fluctuator and the conducting channels.
\end{abstract}

PACS numbers: 73.23.-b, 71.10.Pm, 72.10.Fk, 73.63.Kv

Interference between possible paths is a fundamental quantum mechanical phenomenon. Its realization via an electronic Mach-Zehnder interferometer (MZI $)^{11}$ and an electronic Fabry-Pérot interferometer ${ }^{2]}$ plays an important role in investigating basic physics phenomena such as the Aharonov-Bohm effect, action-free measurement, correlations among particles, and fractional statistics of Abelian and non-Abelian anyons. Understanding the degradation of quantum interference (dephasing) is crucial for nanoelectronic technologies, and is of profound importance for clarifying the elusive transition from a quantum behavior to a classical one. Dephasing of interferometry signal due to the interaction with a detector is intimately related to the entanglement between them. It is often associated with acquiring information about which path the interfering particle has taken $\cdot \frac{3}{6}$

It is common practice to model the effect of an environment on a system through a bath of harmonic oscillators or a puddle of Fermi liquid coupled to the latter. However, recent developments in experimental techniques have made it possible to perform controlleddephasing experiments, where the dephasor consists of only few degrees of freedom, and can be controlled with high precision. This has introduced the need for models of dephasors that are non-Gaussian and fully quantum mechanical. An out-of-equilibrium detector capable of determining the path taken by the electron, and thereby destroying the interference signal, is a standard paradigm in mesoscopic physics. Recently it has been shown that the Friedel sum rule is a useful tool for analyzing dephasing induced by charge fluctuations ${ }^{7}$.

Here we study a paradigmatic controlled-dephasing setup of a MZI interacting with a fluctuator, but with a twist: the fluctuator operates in the fractional quantum Hall regime. The fluctuator is realized by a quantum dot (QD) (cf. Fig. 1), which is electrostatically coupled to the upper arm of the MZI. Since the QD has two charging states ("empty" and "occupied"), the fluctuation pattern of the charge on it defines quantum telegraph noise. Consequently, the phase accumulated along the upper arm of the MZI fluctuates between two val-

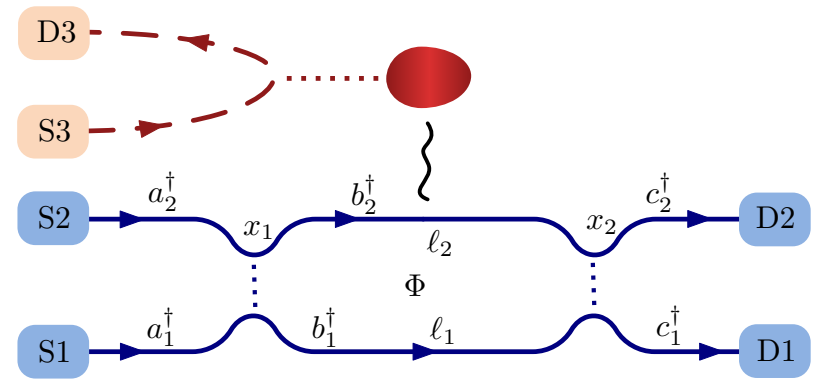

FIG. 1. Schematics of the setup. A quantum dot (QD) (red solid puddle) is capacitively coupled (black wiggly line) to an arm of a Mach-Zehnder interferometer (MZI) (blue solid lines). The QD is tunnel-coupled (red dots) to a chiral Luttinger liquid lead (red dashed line). Tunneling between the two MZI arms is denoted by blue dotted lines. Magnetic flux $\Phi$ is enclosed by the "lower" and "upper" MZI arms whose lengths are $\ell_{1}$ and $\ell_{2}$, respectively. The arrows denote the direction of the chiral motion from the sources ("S") towards the drains ( "D"). Creation operators associated with the various regions of each channel are designated in the figure.

ues $(\theta$ and $\theta+\delta)$. The QD is weakly tunnel-coupled to a channel that supports fractional charge. This is to be contrasted with previous studies ${ }^{7}[10$, which examined a QD that was tunnel-coupled to regular electron reservoirs. We find that: (i) When the gate voltage applied on the QD is varied smoothly, a sharp jump in the phase of the transmission amplitude through the upper arm of the MZI occurs (cf. Fig. 22). (ii) For a symmetric arrangement of the $\mathrm{QD}(\delta=\pi$, see below), the abrupt phase lapse is $\pi$. (iii) Unlike the integer case, even for $\delta \neq \pi$ (asymmetric arrangement), a sharp phase lapse will appear, which differs from $\pi$. (iv) The visibility of the MZI is minimal at the point where a phase lapse occurs, but generically it cannot be fully suppressed. (v) The visibility is a monotonically decreasing function of the entanglement between the MZI and the QD (cf. Fig. 3). The protection against full dephasing is a consequence of the inability of the MZI and the QD to fully entangle.

Consider a quantum Hall state supporting two co- 
propagating edge modes, for instance, filling factor $\nu=$ $4 / 3$. These two edge modes can be utilized for constructing a MZI, capacitively coupled to a nearby QD 10 . Fig. 1 shows schematically such a setup. The MZI consists of two interfering chiral edges - associated with the edge modes of the first fully occupied Landau level - enclosing an Aharonov-Bohm flux, $\Phi$. The QD is tunnel-coupled to another channel associated with the edge modes of the second Landau level. In our analysis, the QD is modeled by a single electronic level, and the tunnel-coupled lead supports fractional charge. Intraand inter-edge interactions within the MZI are neglected.

The interaction between an electron passing through the MZI and the charge on the QD is important. Its effect is to provide the partial wave passing through the upper MZI arm with an extra phase shift $\delta$ when the QD is occupied. This extra phase is a consequence of the variation of the Fermi sea at the upper arm of the MZI due to screening of the QD's potential. Specifically, it was shown ${ }^{7}$ that $\delta / 2 \pi$ is the fraction of electron charge screened by the upper MZI arm when the QD is occupied by one electron. The rest of the electron's charge is screened by other metallic gates/leads, which are not part of our model. Thus, fluctuations of the charge residing on the QD are accompanied by fluctuations in the transmission phase, which act towards dephasing the interference signal of the MZI.

To evaluate the visibility and the transmission phase through the upper MZI arm, which are experimentally measurable quantities, we solve the scattering problem for an electron injected at one of the MZI sources. To this end we define creation operators associated with each of the three regions of the MZI channels (cf. Fig. 1). These are $a^{\dagger}, b^{\dagger}$ and $c^{\dagger}$ for $x<x_{1}, x_{1}<x<x_{2}$ and $x>x_{2}$, respectively. Operators associated with the lower (upper) arm are denoted by the subscript 1 (2). Scattering at the two junctions of the MZI enable tunneling between the channels. The relation between the creation operators in the three regions is given by $\left(b_{1}^{\dagger} b_{2}^{\dagger}\right)=\left(a_{1}^{\dagger} a_{2}^{\dagger}\right) s_{1}^{\dagger}$ and

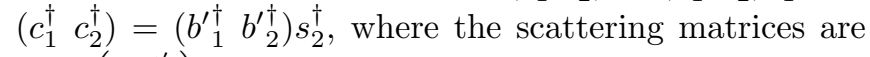
$s_{i}=\left(\begin{array}{cc}r_{i} & t_{i}^{\prime} \\ t_{i} & r_{i}^{\prime}\end{array}\right)$ with $i=1,2$. Primes in the second relation denote that additional phases are accumulated between the two junctions (see below). Note that the operator $b_{2}^{\dagger}$ creates a state before the possible interaction with the QD.

In reality, the initial state of the subsystem composed of the QD and its tunnel-coupled lead may be a very complicated many-body wave function, since the second Landau level is partially filled. However, the MZI interacts electrostatically only with the QD, and not with the electrons residing in the tunnel-coupled lead. Therefore, the only relevant quantity is the part of the wave function that is localized within the QD. This allows us to employ an effective description, whereby the wave function of the QD is that of an isolated two-state system, i.e. a qubit. Any state of this qubit can be written as $|\mathrm{QD}\rangle=e^{i \alpha_{1}} \sqrt{n}|1\rangle+e^{i \alpha_{2}} \sqrt{1-n}|0\rangle$. Here $|1\rangle=d^{\dagger}|0\rangle$, where $d^{\dagger}$ denotes the creation operator of the QD's electronic state, and $\alpha_{1}, \alpha_{2}$ are phases. The probability of the QD to be occupied, $n$, equals the average occupation of the QD. Formally, $n=\left\langle\mathrm{GS}\left|d^{\dagger} d\right| \mathrm{GS}\right\rangle$, where $|\mathrm{GS}\rangle$ denotes the ground state of the QD and its tunnel-coupled lead. The QD's level has energy $\varepsilon$, which is measured relative to the Fermi energy and can be controlled by a gate voltage. In what follows, we treat the QD+lead subsystem as a non-dynamical environment 11 , namely the occupation of the QD is unchanged during the passage of an electron through the upper MZI arm. This assumption is applicable when the time-of-flight through the MZI is much shorter than the typical time of charge fluctuations in the QD. This regime is realized, e.g. in Ref. [10. In addition, we use the inert band approximation and do not consider the renormalization of the tunnel coupling between QD and Luttinger liquid due to the Coulomb interaction with the MZI.

We now turn to solving the scattering problem. Suppose that current is injected at S2 into the upper arm of the MZI. The wave function of the composite system for $x<x_{1}$ is

$$
\left|\chi_{a}\right\rangle=\left(e^{i \alpha_{1}} \sqrt{n} d^{\dagger}+e^{i \alpha_{2}} \sqrt{1-n}\right) a_{2}^{\dagger}|\mathrm{g}\rangle,
$$

where $|\mathrm{g}\rangle$ is the ground state of the MZI+qubit composite. Using the scattering matrix $s_{1}$, one can express $a_{2}^{\dagger}$ in terms of $b_{1}^{\dagger}$ and $b_{2}^{\dagger}$, and thus write the wave function for $x=x_{1}+0$.

The phase accumulated along the lower (upper) arm due to the optical path $\ell_{1}\left(\ell_{2}\right)$ is denoted by $\phi_{\mathrm{d}(\mathrm{u})}$. The Aharonov-Bohm phase $\phi_{\mathrm{AB}}=2 \pi \Phi / \Phi_{0}$, where $\Phi_{0}$ is the magnetic flux quantum, is ascribed to the lower MZI arm. We also recall that, if the QD is occupied, the extra phase accumulated along the upper MZI arm is $\delta$. Thus, the wave function of the entire system corresponding to an electron at $x=x_{2}-0$ is

$$
\begin{aligned}
\left|\chi_{b}\right\rangle & =e^{i\left(\alpha_{1}+\phi_{\mathrm{d}}+\phi_{\mathrm{AB}}\right)}\left\{\sqrt{n}\left[t_{1}^{\prime} d^{\dagger}{b^{\prime}}_{1}^{\dagger}+r_{1}^{\prime} e^{i\left(\phi_{\mathrm{u}}-\phi_{\mathrm{d}}-\phi_{\mathrm{AB}}+\delta\right)} d^{\dagger} b_{2}^{\prime \dagger}\right]\right. \\
& \left.+\sqrt{1-n} e^{i\left(\alpha_{2}-\alpha_{1}\right)}\left[t_{1}^{\prime}{b^{\prime}}_{1}^{\dagger}+r_{1}^{\prime} e^{i\left(\phi_{\mathrm{u}}-\phi_{\mathrm{d}}-\phi_{\mathrm{AB}}\right)} b_{2}^{\prime \dagger}\right]\right\}|\mathrm{g}\rangle \cdot(2)
\end{aligned}
$$

Employing the scattering matrix $s_{2}$ one finally obtains the wave function of the entire system with $x=x_{2}+0$,

$$
\begin{aligned}
\left|\chi_{c}\right\rangle & =e^{i\left(\alpha_{1}+\phi_{\mathrm{d}}+\phi_{\mathrm{AB}}\right)}\left(\hat{A} c_{1}^{\dagger}+\hat{B} c_{2}^{\dagger}\right)|\mathrm{g}\rangle, \\
\hat{A} & =\left[-\sqrt{n} d^{\dagger}+\sqrt{1-n} e^{i\left(\alpha_{2}-\alpha_{1}\right)}\right] t_{1}^{\prime} r_{2} \\
& +e^{i \alpha_{3}}\left[-\sqrt{n} e^{i \delta} d^{\dagger}+\sqrt{1-n} e^{i\left(\alpha_{2}-\alpha_{1}\right)}\right] r_{1}^{\prime} t_{2}^{\prime}, \\
\hat{B} & =\left[-\sqrt{n} d^{\dagger}+\sqrt{1-n} e^{i\left(\alpha_{2}-\alpha_{1}\right)}\right] t_{1}^{\prime} t_{2} \\
& +e^{i \alpha_{3}}\left[-\sqrt{n} e^{i \delta} d^{\dagger}+\sqrt{1-n} e^{i\left(\alpha_{2}-\alpha_{1}\right)}\right] r_{1}^{\prime} r_{2}^{\prime},
\end{aligned}
$$

where $\alpha_{3} \equiv \phi_{\mathrm{u}}-\phi_{\mathrm{d}}-\phi_{\mathrm{AB}}$. The current at D1 is proportional to the probability of finding there the electron,

$$
T=\left|t_{1}^{\prime} r_{2}\right|^{2}+\left|r_{1}^{\prime} t_{2}^{\prime}\right|^{2}+2\left|t_{1}^{\prime} r_{2} r_{1}^{\prime} t_{2}^{\prime}\right| \Re\left[e^{i \alpha_{4}}\left\langle e^{i \tilde{\delta}}\right\rangle\right],
$$


with $\alpha_{4} \equiv \phi_{\mathrm{u}}-\phi_{\mathrm{d}}-\phi_{\mathrm{AB}}+\beta_{2}-\beta_{1}$, and the phases $\beta_{1}$ and $\beta_{2}$ are defined by $t_{1}^{\prime} r_{2} \equiv\left|t_{1}^{\prime} r_{2}\right| e^{i \beta_{1}}$ and $r_{1}^{\prime} t_{2}^{\prime} \equiv\left|r_{1}^{\prime} t_{2}^{\prime}\right| e^{i \beta_{2}}$. We have abbreviated $n e^{i \delta}+(1-n)$ as $\left\langle e^{i \tilde{\delta}}\right\rangle$. Hereafter $\langle\cdots\rangle$ denotes averaging with respect to the probability distribution function

$$
P(\tilde{\delta})= \begin{cases}n & \text { for the phase to be } \delta \\ 1-n & \text { for the phase to be } 0\end{cases}
$$

The parameter $\delta$ is defined above, and a corresponding random variable is denoted by $\tilde{\delta}$. Thus, it is obtained that the part of the transmission phase acquired due to the interaction with the QD is a statistical variable whose probability distribution is determined by $n$ (cf. Ref. 11).

From Eq. (4) we obtain the transmission phase through the upper MZI arm and the visibility,

$$
\begin{aligned}
\arg \left\langle e^{i \tilde{\delta}}\right\rangle & =\arg \left[n\left(e^{i \delta}-1\right)+1\right] \\
v & =\frac{2}{p^{-1}+p}\left|\left\langle e^{i \tilde{\delta}}\right\rangle\right|=\frac{2\left|1+n\left(e^{i \delta}-1\right)\right|}{p^{-1}+p}
\end{aligned}
$$

with $p=\left|r_{1}^{\prime} t_{2}^{\prime} / t_{1}^{\prime} r_{2}\right|$.

Several observations can be extracted from the results outlined above. Full dephasing requires $\delta=\pi$, as well as $n=1 / 2$ for a particular value of $\varepsilon$. Moreover, the visibility $v(n)$ is symmetric with respect to $n=1 / 2$, and is thus continuous at that point, even if $n(\varepsilon)$ is not. As long as $\delta \neq 0$ the point $n=1 / 2$ is the minimum of $v(n)$ (reaching 0 for $\delta=\pi$ ), and $v(n)$ increases monotonically when $n$ moves away from that point (when $\delta=0$ it follows that $v(n)=1$ ). In particular, if for some reason the occupation of the QD cannot be $1 / 2$, then the visibility cannot reach zero, namely the MZI cannot be fully dephased.

To complete the calculation of the transmission phase and the visibility one needs to substitute the appropriate function for $n(\varepsilon)$. A QD tunnel-coupled to a TomonagaLuttinger liquid is analysed in Ref. 12, where a perturbative calculation in the QD-lead tunneling matrix element yields $n(\varepsilon>0)=\left[\Gamma / \pi \rho_{1}(0)\right] \int_{0}^{\infty} \mathrm{d} \omega \rho_{g}(\omega) /(\omega+\varepsilon)^{2}$. Here $\rho_{g}(\omega)$ is the tunneling density of states of the TomonagaLuttinger liquid, and $g$ is the Luttinger parameter. If $g=1$, i.e. in the Fermi liquid case, $\Gamma$ takes the physical meaning of the level width. In our case $g \rightarrow \nu-1$, where $\nu$ is the filling factor in the bulk. For $\varepsilon<0$ electron-hole symmetry implies $n(\varepsilon)=1-n(-\varepsilon)$. It follows that $n(\varepsilon)$ is a monotonically decreasing function, and that $\mathrm{d} n / \mathrm{d} \varepsilon$ is a symmetric function. The intriguing feature of $n(\varepsilon)$ is that the QD occupancy jumps abruptly at $\varepsilon=0$ downwards if $\Gamma$ is sufficiently small (see below). This phenomenon has to do with the suppression of tunneling into a Tomonaga-Luttinger liquid at low energies, which gives rise ${ }^{\sqrt{12}}$ to a renormalized level width, and under certain circumstances may result in a degenerate ground state of the QD+lead system. The abrupt jump in $n(\varepsilon)$ implies that the value $n=1 / 2$ is inaccessible as $\varepsilon$ is varied. Consequently, full dephasing is not possible, even when $\delta=\pi$, cf. Eq. (6b). Furthermore, according to Eq. (6a)

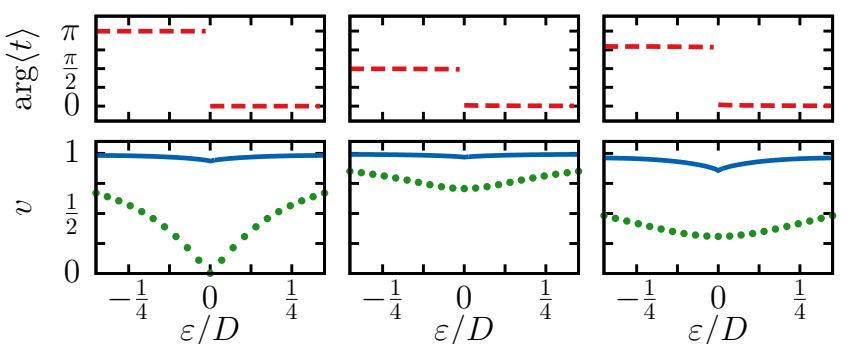

FIG. 2. Transmission phase through the upper MZI arm (red dashed lines, top) and its visibility (blue solid lines, bottom) as a function of the dimensionless energy parameter $\varepsilon / D$ for the fractional case. The visibility in the integer case is also shown (green dotted lines, bottom). In the fractional case the visibility cannot vanish, but a phase lapse always occurs. In the integer case the visibility can vanish, and only then a phase lapse occurs. Here $\Gamma / D=0.2, \delta=\pi$ (left); $\Gamma / D=0.2$, $\delta=\pi / 2$ (center); $\Gamma / D=0.5, \delta=0.8 \pi$ (right); and $p=1$ everywhere.

the abrupt jump in $n(\varepsilon)$ is accompanied by an abrupt phase jump.

As an example, we focus on the case of $\nu=4 / 3$, where the MZI consists of the chiral inner edge channels associated with the first full Landau level, and the QD is coupled to the outer edge channel associated with the second, $1 / 3$-filled, Landau level. Under these circumstances ${ }^{12}$, $n(\varepsilon>0) \simeq(\Gamma / 2 \pi D)[1+2(\varepsilon / D) \ln (\varepsilon / D)]$, where $D$ is the bandwidth in the Tomonaga-Luttinger liquid. It follows that $n(\varepsilon)$ is discontinuous at $\varepsilon=0$; the downwards discontinuity is $1-\Gamma / \pi D$. It is accompanied by a sharp jump in the transmission phase through the upper MZI arm whose magnitude is

$$
\begin{aligned}
& \arg \left\langle e^{i \tilde{\delta}}\right\rangle_{0^{+}}-\arg \left\langle e^{i \tilde{\delta}}\right\rangle_{0^{-}}=\arg [1-\Gamma / 2 \pi D \\
& \left.+(\Gamma / 2 \pi D) e^{i \delta}\right]-\arg \left[\Gamma / 2 \pi D+(1-\Gamma / 2 \pi D) e^{i \delta}\right] .
\end{aligned}
$$

Since $n \neq 1 / 2$, the visibility is always finite. The transmission phase and visibility $\underline{13}$ are depicted in Fig. 2 .

To emphasize the uniqueness of our results, we contrast them with a more trivial scenario, where the channel tunnel-coupled to the QD supports an integer filling factor. Equations (6a) and (6b) for the transmission phase and visibility remain valid, with the proviso that the occupation of the QD is given by $14(\varepsilon)=$ $[\pi / 2-\arctan (\varepsilon / \Gamma)] / \pi$. Substitution in Eq. $6 \mathrm{~b}$ readily gives $\left|\left\langle e^{i \tilde{\delta}}\right\rangle\right|=\left|1+\left(e^{i \delta}-1\right)[\pi / 2-\arctan (\varepsilon / \Gamma)] / \pi\right|$, which is also depicted in Fig. 2. As $n$ increases from 0 to 1, the transmission phase given by Eq. 6a increases (decreases) continuously from 0 to $\delta(2 \pi$ to $\delta)$ if $\delta<\pi$ $(\delta>\pi)$. Only for $\delta=\pi$ there is an abrupt jump in the transmission phase, whose magnitude is $\pi$, at $n=1 / 2$ (the direction of the jump is undefined). The visibility is a symmetric function with respect to $\varepsilon=0$, and has a minimum at that point. If $\delta=\pi$, the visibility vanishes at $\varepsilon=0$, where $n=1 / 2$. Thus, in the integer case a phase lapse is generically absent, and full dephasing may occur. We note that this result can be also obtained by calculating a certain overlap integral associated with the 
QD+lead wave function ${ }^{7}$. The correspondence between this approach and the one we used is another manifestation of two viewpoints vis-à-vis dephasing 11 .

What is the physical origin of the striking behaviour of the visibility in the fractional case as compared to the integer case? As we shall see, it is the degree of entanglement between the QD and the MZI. We quantify this entanglement with the help of the concurrence ${ }^{15} C$. In the concerned setup the concurrence is a measure of the entanglement, extracted from the density matrix of the system. The latter includes all the information about the state of the system, and in particular on the entanglement between its constituents. Larger values of the concurrence signify larger entanglement in the system, where $C=0(1)$ implies no (complete) entanglement. Using Eq. (2) and the definition of the concurrence ${ }^{15}$, one obtains

$$
C(n, \delta)=4 \sqrt{n(1-n)}\left|t_{1}^{\prime}\right| \sqrt{1-\left|t_{1}^{\prime}\right|^{2}}|\sin (\delta / 2)|,
$$

which is plotted in Fig. 3 (inset). The concurrence (entanglement) is proportional to the quantum fluctuations in the occupation of the QD and to the quantum fluctuations in the occupation of the MZI's arm. It has a maximum at $(n, \delta)=(1 / 2, \pi)$, the point of full dephasing.

In fact, it is possible to express the visibility as a function of the concurrence. This is given by

$$
v(C)=2\left(p+p^{-1}\right)^{-1}\left[1-C^{2} / 4\left|t_{1}^{\prime}\right|^{2}\left(1-\left|t_{1}^{\prime}\right|^{2}\right)\right]^{\frac{1}{2}},
$$

and is shown in Fig. 3. This relation has a clear physical meaning: the larger the entanglement between the MZI and the QD, the larger is the dephasing. Taking the opposite point of view, we can say that it is possible to measure the entanglement between the two subsystems by measuring the visibility of the interferometer. This demonstrates the intimate relation between the visibility of the interferometer and the ability of an interfering electron to entangle to an electron residing on the QD.

The importance of the results reported here is twofold. From a fundamental point of view, we note that for a given value of $\delta$ the interaction between the interferometer and the QD is identical in the fractional and integer cases. Nevertheless, the dephasing of the interferometer by the QD+lead system - and hence the entanglement between them - is utterly different. This clearly underlines the importance of the internal dynamics of a system in the process of entangling to another system. From the experimental point of view, the sharp jump in the phase accompanied by high visibility can be used to identify unequivocally Luttinger liquid physics. Such a measurement is within reach 10 , and the pronounced features should be easy to observe. We also stress that the system considered here can be realized in other contexts, e.g. with carbon nanotubes, where the MZI is replaced by a relatively open Fabry-Pérot interferometer (so as to suppress multiple windings).

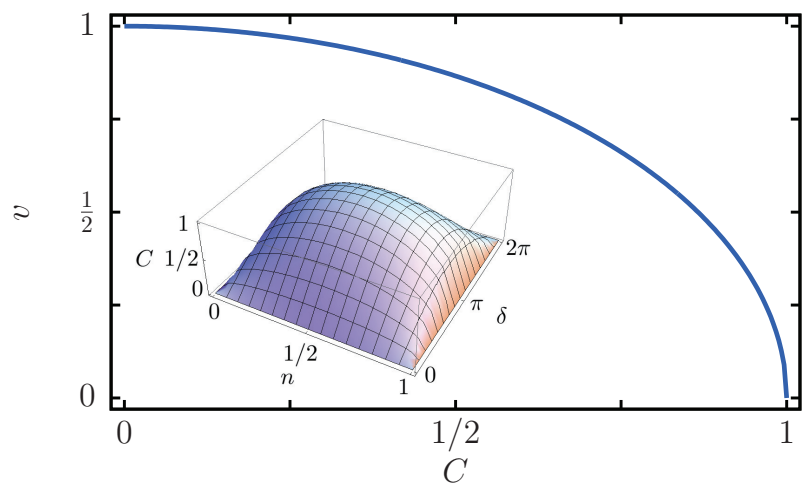

FIG. 3. The visibility as a function of the concurrence, $v(C)$, is a monotonically decreasing function (cf. Eq. (9)). Since, roughly, the concurrence is a measure of the entanglement between an electron passing through the MZI and an electron residing on the $\mathrm{QD}$, this shows that the larger the entanglement between them, the larger is the dephasing of the MZI. Inset: The concurrence $C$ as a function of the localized state occupation $n$ and the screening parameter $\delta$ (cf. Eq. (8)). The maximum concurrence at $(n, \delta)=(1 / 2, \pi)$ signifies maximum (and complete) entanglement between an electron interfering through the MZI and an electron residing on the QD. In both graphs $\left|t_{1}^{\prime}\right|=\left|t_{2}^{\prime}\right|=1 / \sqrt{2}$.

To conclude, we have analysed the interference degradation of a MZI due to coupling to a QD which serves as a generator of quantum telegraph noise. Charge fluctuations in the QD due to tunnel-coupling to a chiral edge state dephase the MZI. The amount of dephasing is related to the degree of entanglement between the MZI and the QD. We have studied the case where the QD is tunnel-coupled to a chiral channel supporting a fractional bulk filling and contrasted it with the case of an integer filling. In the former case, when the edge channel is modeled by a chiral Luttinger liquid, the visibility cannot vanish, and a phase lapse always occurs. In the integer case the visibility can vanish, and a phase lapse occurs only under strict symmetry conditions. This reduction of dephasing is shown to be intimately related to the fact that the system (MZI) and the detector (QD) cannot be fully entangled.

We are grateful to Y. Ronen, H. K. Choi, E. Weisz, Y. E. Kraus, and M. Heiblum for useful discussions. Financial support by the German-Israel Foundation (GIF), DFG grant RO 2247/8-1, and the Israel Science Foundation is acknowledged. 
1 Y. Ji, Y. Chung, D. Shprinzak, M. Heiblum, D. Mahalu, and H. Shtrikman, Nature (London) 422, 415 (2003); I. Neder, M. Heiblum, Y. Levinson, D. Mahalu, and V. Umansky, Phys. Rev. Lett. 96, 016804 (2006); P. Roulleau, F. Portier, D. C. Glattli, P. Roche, A. Cavanna, G. Faini, U. Gennser, and D. Mailly, Phys. Rev. B 76, 161309(R) (2007); L. V. Litvin, H.-P. Tranitz, W. Wegscheider, and C. Strunk, ibid. 75, 033315 (2007); L. V. Litvin, A. Helzel, H.-P. Tranitz, W. Wegscheider, and C. Strunk, ibid. 78, 075303 (2008); P. Roulleau, F. Portier, P. Roche, A. Cavanna, G. Faini, U. Gennser, and D. Mailly, Phys. Rev. Lett. 100, 126802 (2008); E. Bieri, M. Weiss, O. Goktas, M. Hauser, C. Schonenberger, and S. Oberholzer, Phys. Rev. B 79, 245324 (2009); P. Roulleau, F. Portier, P. Roche, A. Cavanna, G. Faini, U. Gennser, and D. Mailly, Phys. Rev. Lett. 102, 236802 (2009).

${ }^{2}$ F. E. Camino, W. Zhou, and V. J. Goldman, Phys. Rev. B 76, 155305 (2007), Y. Zhang, D. T. McClure, E. M. Levenson-Falk, C. M. Marcus, L. N. Pfeiffer, and K. W. West, Phys. Rev. B 79, 241304 (2009) N. Ofek, A. Bid, M. Heiblum, A. Stern, V. Umansky, and D. Mahalu, Proceedings of the National Academy of Sciences 107, 5276 (2010); D. T. McClure, W. Chang, C. M. Marcus, L. N. Pfeiffer, and K. W. West, Phys. Rev. Lett. 108, 256804 (2012)

${ }^{3}$ I. Neder, M. Heiblum, D. Mahalu, and V. Umansky, Phys. Rev. Lett. 98, 036803 (2007); I. Neder, F. Marquardt,
M. Heiblum, D. Mahalu, and V. Umansky, Nat. Phys. 3, 534 (2007).

${ }^{4}$ Y. Levinson, Europhys. Lett. 39, 299 (1997).

5 I. L. Aleiner, N. S. Wingreen, and Y. Meir, Phys. Rev. Lett. 79, 3740 (1997).

6 S. A. Gurvitz, Phys. Rev. B 56, 15215 (1997)

7 B. Rosenow and Y. Gefen, Phys. Rev. Lett. 108, 256805 (2012)

${ }^{\circ}$ A. Grishin, I. V. Yurkevich, and I. V. Lerner, Phys. Rev. B 72, 060509 (2005); I. V. Yurkevich, J. Baldwin, I. V. Lerner, and B. L. Altshuler, Phys. Rev. B 81, 121305 (2010)

${ }^{9}$ I. Neder and F. Marquardt, New J. Phys. 9, 112 (2007); B. Abel and F. Marquardt, Phys. Rev. B 78, 201302 (2008)

io E. Weisz, H. K. Choi, M. Heiblum, Y. Gefen, V. Umansky, and D. Mahalu, Phys. Rev. Lett. 109, 250401 (2012).

11 A. Stern, Y. Aharonov, and Y. Imry, Phys. Rev. A 41, $3436(1990)$.

${ }_{12}$ A. Furusaki and K. A. Matveev, Phys. Rev. Lett. 88, 226404 (2002).

13 See Supplemental Material for further discussion of the transmission probability through the MZI, Eq. (4).

14 G. D. Mahan, Many-Particle Physics, 3rd ed. (Kluwer Academic / Plenum Publishers, New York, 2000) Chap. 4.2.

15 W. K. Wootters, Phys. Rev. Lett. 80, 2245 (1998). 


\section{SUPPLEMENTAL MATERIAL}

\section{The transmission probability through the Mach-Zehnder interferometer}

In the main text we discuss the visibility of the interference signal through the Mach-Zehnder interferometer (MZI) and the transmission phase through its "upper" arm. Both these quantities can be extracted from the transmission probability given by Eq. (4) in the main text. These are the physically relevant quantities, since they are independent of the specific value of the Aharonov-Bohm flux $\Phi$, which serves as a means of measuring the transmission phase. Here, we provide further information on Eq. (4), which may facilitate an experimental investigation of the setup.

Eq. (4) presents the transmission probability through the MZI, which is directly measured experimentally. It depends on the scattering matrices of the junctions of the MZI, as well as on the phase $\alpha_{4}$ (see text after Eq. (4)). However, the physics referred to in the main text is essentially given by $\left\langle e^{i \tilde{\delta}}\right\rangle$. Thus, in what follows we take $\left|t_{1}^{\prime} r_{2}\right|=\left|r_{1}^{\prime} t_{2}^{\prime}\right|=1 / 2$ and $\alpha_{4}=0$, which yield

$$
T=\frac{1}{2}+\frac{1}{2}\left|\left\langle e^{i \tilde{\delta}}\right\rangle\right| \cos \left(\arg \left\langle e^{i \tilde{\delta}}\right\rangle\right)
$$

This is depicted in Fig. I.1 for the three cases shown in Fig. (2), both for the fractional and integer regimes.

As can be seen from Fig. I.1. and as can be also verified by Eq. (4), an abrupt jump in the transmission phase leads to an abrupt jump in the transmission probability through the MZI. Thus for the integer case the transmission probability is continuous (when $\delta=\pi$ the transmission phase jumps abruptly, but at the same point the visibility vanishes, thus rendering the jump invisible). In contrast, in the fractional case the transmission probability always jumps abruptly.
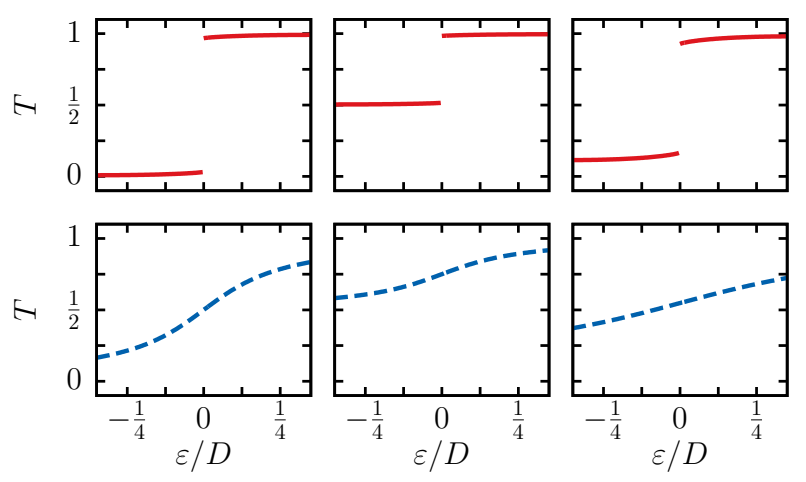

FIG. I.1. The transmission probability through the MachZehnder interferometer as a function of the dimensionless energy parameter $\varepsilon / D$ for the fractional case (upper panel) and integer case (lower panel), see Eq. (I.1). The abrupt jump is the hallmark of the fractional case. Here $\Gamma / D=0.2, \delta=\pi$ (left); $\Gamma / D=0.2, \delta=\pi / 2$ (center); $\Gamma / D=0.5, \delta=0.8 \pi$ (right); $\left|t_{1}^{\prime} r_{2}\right|=\left|r_{1}^{\prime} t_{2}^{\prime}\right|=1 / 2$ and $\alpha_{4}=0$ everywhere.

We have focused on the transmission probability from S2 to D1. We note that other transmission probabilities may be calculated similarly, i.e. the transmission probabilities from S1 to D1, from S1 to D2, and from S2 to D2. However, the same physics discussed here for the pair S2-D1 holds for other pairs. So considering, for example, the transmission probability from S2 to D2, does not add any qualitatively new information. 